\title{
EDUCACIÓN, NACIÓN Y TECNOLOGÍA
}

Cremaschi Gustavo

Cremaschi María Elisa, Raúl Barandiarán

Laboratorio de Tecnología y Gestión Habitacional, Facultad de Arquitectura y Urbanismo, Universidad Nacional de La Plata, La Plata, Bs.As., Argentina, 47 N 162,

latec@fau.unlp.edu.ar, elisacre@hotmail.com

Palabras clave: Tecnología - Innovación Curricular - Prácticas Pre Profesionales

\section{Resumen:}

En el marco del IX Congreso Regional de Tecnología de la Arquitectura cuyo tema es "Arquitectura, Diseño y Tecnología en la Construcción Sostenible del Ambiente" resulta de interés compartir las estrategias pedagógicas y herramientas didácticas implementadas en las Prácticas Pre Profesionales Asistidas (PPPA) desarrolladas en el marco del Plan de Estudios VI a través del programa de Vinculación Tecnológica de la Facultad de Arquitectura y Urbanismo de la Universidad Nacional de La Plata. El programa tiene lugar físico dentro de un predio de carácter universitario con varios galpones longevos pertenecientes a distintas unidades académicas que requieren de propuestas fehacientes de recuperación. Su rehabilitación es imprescindible para afrontar los desafíos de los años por venir y los avances que necesariamente deben desarrollarse en la industria de la construcción en comparación con los avances de otras industrias, tanto en cuestiones como el ahorro energético, el cuidado del medio ambiente, la integración social y el progreso económico de nuestra sociedad, que son las bases del desarrollo sustentable.

Ante lo dicho y bajo la necesidad imperiosa de crear un Espacio de Investigación Aplicada en la Construcción, en donde se puedan ensayar nuevas tecnologías, probar propuestas de investigación aplicada y contribuir al progreso técnico, se implementaron como parte del Programa de Vinculación Tecnológica (PVT), PPPA para cuali - cuantificar las tareas necesarias para la rehabilitación del galpón FAU en pos de aportar a la formación integral del estudiante de arquitectura, sumando saberes de grado, investigación y vinculación con el medio.

\section{Introducción.}

El crecimiento de la población y el desarrollo tecnológico de nuestros tiempos han originado novedosas formas de confort para los habitantes, lo cual a su vez se traduce en mayor variedad de instalaciones y equipos, $y$, en consecuencia, en una demanda creciente de energía difícilmente satisfecha por la oferta convencional. Estudios y aproximaciones recientes - como las conclusiones de la Cumbre de Río en junio de 1992, el Protocolo de Kyoto en diciembre de 1997 y la Cumbre de Johannesburgo en 2002- han identificado la necesidad de reordenar y repensar el consumo de energía en el mundo, para reducir así las emisiones de gases al ambiente; también se ha concentrado la mirada en el tema de las edificaciones, responsables del consumo de alrededor de $40 \%$ de energía en las ciudades. En consecuencia, disciplinas como la Arquitectura e Ingeniería se han visto en la necesidad de repensarse en una mayor armonía con el medio ambiente y en consecuencia una reducción del consumo de energía.

En el marco de la educación universitaria actual, la Universidad Nacional de La Plata (UNLP) se desarrolla bajo un gran esfuerzo entre incertidumbres y utopías dentro de un contexto de crisis mundial generalizada con especial impacto en América Latina y Argentina. Esta precarización de las condiciones en que se encuentran las instituciones en general $y$, en particular la que nos compete, conlleva el desafío de construir alternativas posibles de transformación.

El Programa de Vinculación Tecnológica tiene como objetivo profundizar la relación e interacción de la Facultad con la producción a través de la relación con el sector público y privado, colaborando en la obtención de nuevos desarrollos y tecnologías, así como la identificación y adaptación de tecnologías disponibles, promoviendo en las Cátedras, Grupos de Investigación e Institutos la 
vocación de participar y relacionarse con el medio. El desarrollo del programa pretende generar un círculo virtuoso entre la Universidad y la sociedad, así como el fortalecimiento de la relación de la Facultad con el medio productivo en general y el regional en particular.

El programa presenta la necesidad imperiosa de poseer un Espacio de Investigación Aplicada en la Construcción, en el que las Cátedras, los Talleres, los extensionistas y las unidades de investigación de la Facultad de Arquitectura y Urbanismo (FAU), tengan un área en el que se puedan ensayar nuevas tecnologías, probar propuestas de investigación aplicada y contribuir al progreso técnico pasible de ser utilizado por la industria a partir de la generación de patentes, permitirá generar un círculo virtuoso entre la Universidad y la sociedad, así como el fortalecimiento de la relación de la Facultad con el medio productivo en general y el regional en particular.

El nuevo espacio, conseguido por la FAU a partir de larguísimas tratativas, tiene lugar físico dentro de un predio de carácter universitario con varios galpones muy antiguos, antes pertenecientes a la Autoridad del Agua de la Provincia de Buenos Aires (Figura 1). Su rehabilitación es imprescindible para afrontar los desafíos de los años por venir y los avances que necesariamente deben desarrollarse en la industria de la construcción en comparación con los avances de otras industrias, tanto en cuestiones como el ahorro energético, el cuidado del medio ambiente, la integración social y el progreso económico de nuestra sociedad, que son las bases del desarrollo sustentable.

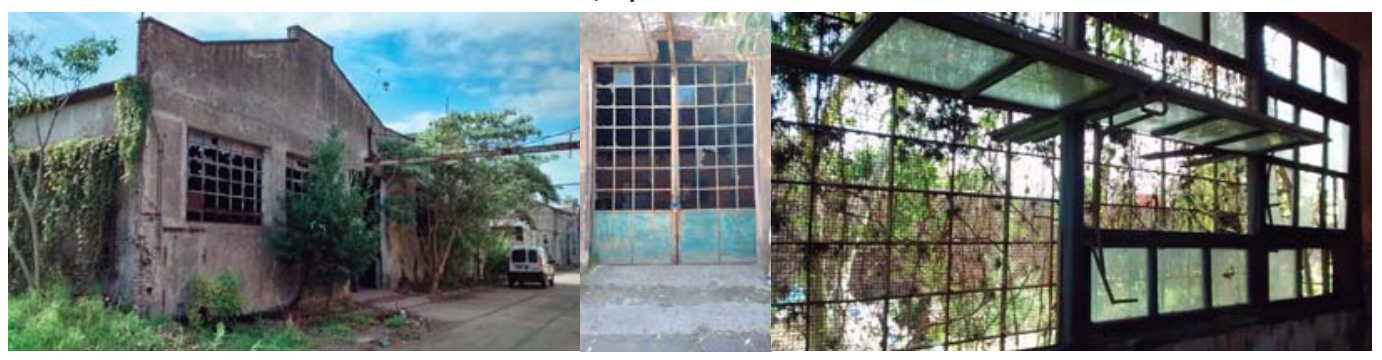

Figura 1. Galpón FAU en Predio Universitario. Fuente: Elaboración propia. 2016.

En este sentido, desde el programa de Vinculación Tecnológica se plantea el reto de desarrollar estrategias y promover nuevas tecnologías que incorporen gradualmente el concepto de Infraestructura Universitaria Sustentable a través de las prácticas pre profesionales asistidas (PPPA) de manera tal de obtener "mejor valor" por el dinero público que se invierte y contribuir en la formación de los futuros graduados profesionales y por ende de la sociedad y de la cultura.

Ante este requerimiento, y frente a los nuevos modos de producción de conocimiento, la interdisciplinariedad, el impacto de las tecnologías de la información y la comunicación; la transformación y heterogeneidad de la matrícula, se diseñaron como parte del programa, PPPA para realizar la rehabilitación e intervención sistémica y sustentable de un edificio universitario a fin de poner en práctica los saberes aprendidos en el currículum de grado y fortalecer la adquisición práctica de los conocimientos formales propios de las incumbencias profesionales. Para que esto sea posible, es necesario que el alumno comprenda la relación entre tecnologías de construcción, uso del edificio, su duración, y su costo de mantenimiento, tanto activo como pasivo, y su vinculación con el concepto de sustentabilidad de la obra de arquitectura.

Estos procesos de innovación en el currículum y en las metodologías de enseñanza se pretenden implementar en edificios institucionales de la UNLP que requieran de propuestas fehacientes de recuperación. La intervención sustentable de la infraestructura universitaria es imprescindible para afrontar los desafíos de los años por venir y los avances que necesariamente deben desarrollarse en la industria de la construcción en comparación con los avances de otras industrias, tanto en cuestiones como el ahorro energético, el cuidado del medio ambiente, la integración social y el progreso económico de nuestra sociedad, que son las bases del desarrollo sustentable. En base a esto y teniendo en cuenta que la tecnología es imprescindible e inevitable, como bien lo afirmaba Jorge Sábato, "el problema no es el de saber si se necesita tecnología sino el de precisar qué tecnología se necesita".

Se toma como premisa que la Arquitectura involucra el proceso desde las ideas iniciales que sustentan las decisiones, hasta la materialización, la calidad de vida generada para el hombre, el 
uso y la evaluación post-ocupacional. Es por ello que la propuesta tiene como visión aportar a la formación de un arquitecto con vocación de servicio, con profundo compromiso social e individual, sensible a las necesidades del hombre, poniendo en consecuencia al servicio del mismo el máximo de su capacidad intelectual, cuidando los recursos que ella tiene y produce. Teniendo en claro que el objetivo no es formar técnicos constructores sino arquitectos con compromiso ambiental y sensibilidad social. Arquitectos capaces de reconocer a la sustentabilidad como un concepto integral que pretende identificar y desarrollar sus ejes compositivos: Sustentabilidad Social, Sustentabilidad Económica, Sustentabilidad Urbana y Sustentabilidad Tecnológica.

\section{Objetivos}

\subsection{Objetivo General}

Poner en práctica los saberes aprendidos por el estudiante en el currículum de grado y fortalecer la adquisición práctica de los conocimientos formales propios de las incumbencias profesionales, a través de la realización del diagnóstico para la reparación, rehabilitación y restauración del nuevo espacio FAU.

\subsection{Objetivos Particulares}

- Proporcionar al alumno una aproximación a la práctica profesional, afianzando y concluyendo el nivel de preparación logrado en el transcurso de la carrera.

- Profundizar el ejercicio del pensamiento crítico y creador, a los efectos de sortear situaciones concretas y objetivas en relación con las amplias problemáticas actuales.

- Orientar adecuadamente al futuro egresado dentro de las perspectivas laborales y de especialización de la profesión en la región.

- Verificar un pensamiento sistémico integrado para la labor profesional

- Aportar a la formación integral del estudiante de arquitectura, sumando saberes de grado, investigación y vinculación con el medio.

\section{$3 \quad$ Metodología}

La metodología de trabajo se construyó en pos de lograr un pensamiento sistémico integrado para la labor profesional y estuvo compuesta por las tareas mínimas necesarias la rehabilitación del edificio.

Se priorizó la utilización de tecnologías apropiadas y transferibles, capaces de posibilitar cambios sustanciales, minimizar el uso de recursos escasos y brindar la resolución de un problema con la mejor relación insumo/producto. Con lo que fue de suma importancia, crear en el alumno la conciencia, que un responsable estudio de la biozona de implantación de la obra, no sólo permitió adoptar las mejores soluciones para enfrentar los desafíos climáticos, sino que permitió analizar el uso favorable de esos factores en pos de una mayor prestación del edificio, apuntando a un menor gasto energético en pos del cuidado del medio ambiente. De esta forma se logró que el alumno experimente en escala 1:1 el camino de la edificación sustentable, estudiando, analizando y aplicando soluciones sustentables en la propuesta de intervención, seleccionando y utilizando tecnologías de bajo impacto ambiental y uso racional de la energía.

Las prácticas estuvieron compuestas por un grupo de 4 (cuatro) alumnos próximos a egresar: Florencia Facenda, Gastón Ferández, Luciano Di Gregorio y Romina Paggis, los cuales trabajaron sobre dos subsistemas, a saber: envolvente vertical e instalaciones, los cuales estuvieron divididos básicamente en: carpinterías y mampostería para el primer caso y en electricidad, baja tensión, gas, agua y cloaca para el segundo caso. Cabe aclarar que el subsistema cubierta fue descartado por cuestiones de seguridad de los alumnos.

Se desarrollaron tres fases de trabajo para poder cumplir el objetivo propuesto: una primera fase de formulación del programa de actividades y recopilación de información necesaria, una segunda de relevamiento y síntesis de la información, y una tercera de propuesta, cómputo y presupuesto: 
FASE 1: Formulación del Programa de Actividades y Recopilación de la Información (Figura 2)

1.1. Visita e identificación del edificio.

1.2. Recopilación y selección de antecedentes en materia de relevamiento de obras.

1.3. Delimitación el área (rubro) de estudio con los tutores.

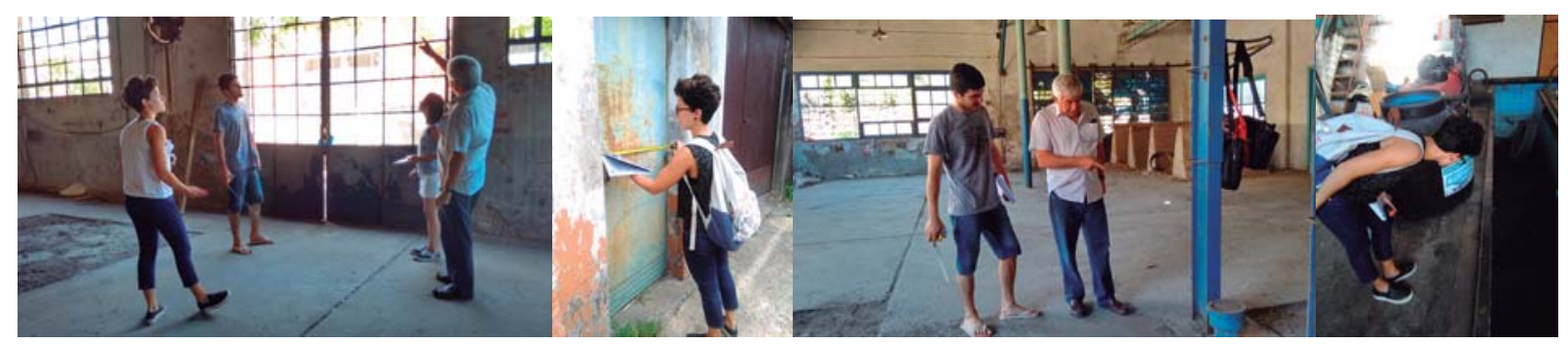

Figura 2. Fase 1: Visita e identificación del edificio y delimitación del área de estudio con los tutores.

Fuente: Elaboración propia. 2016.

FASE 2: Relevamiento y Síntesis de la Información (Figura 3)

2.1. Relevamiento del edificio y registro del subsistema designado por el tutor. (Características del edificio, definición constructiva, estado patológico, entre otros).

2.2. Análisis de los rubros relevados y elaboración de fichas síntesis

2.3. Planteo de soluciones y alternativas de reparación.

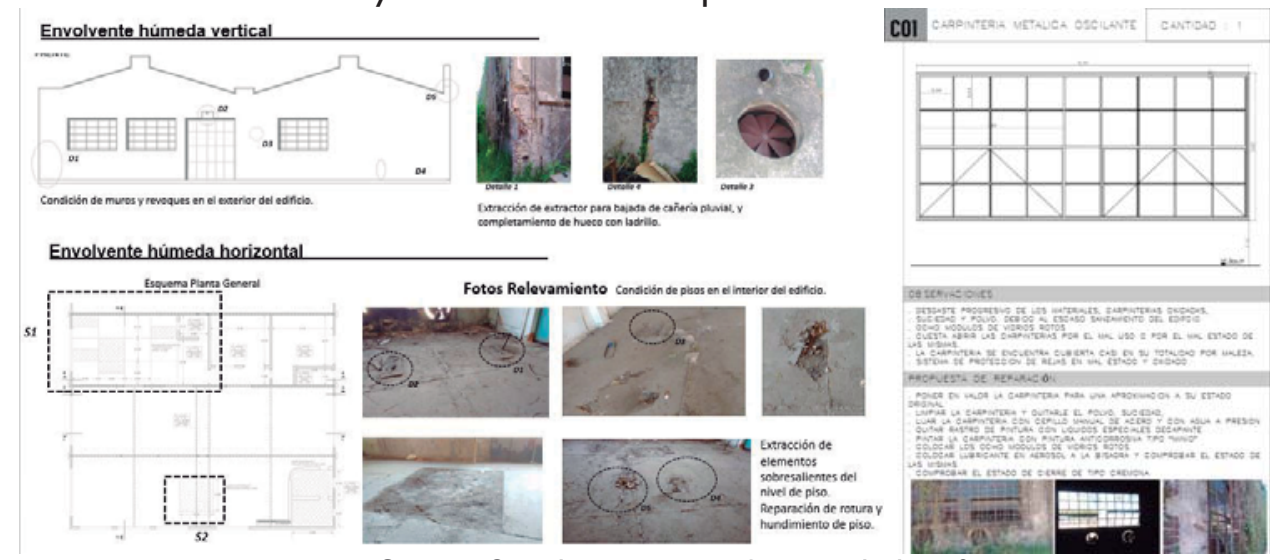

Figura 3. Fase 2: Relevamiento y Síntesis de la Información.

Fuente: Elaboración propia. 2017.

\section{FASE 3: Propuesta, Cómputo y Presupuesto (Figura 4)}

3.1. Diseño de la propuesta del subsistema asignado y elaboración de documentación necesaria.

3.2. Cómputo de materiales y elaboración de presupuesto.

3.3. Elaboración de la memoria técnica.

3.4. Elaboración del informe final.
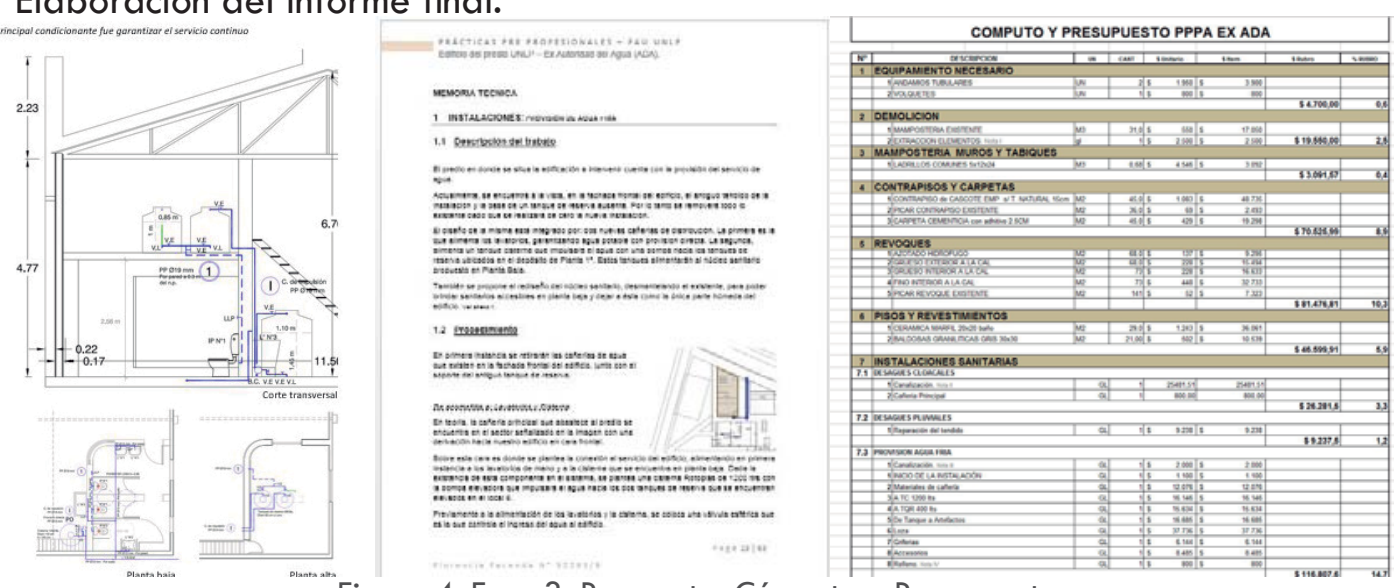

Figura 4. Fase 3: Propuesta, Cómputo y Presupuesto

Fuente: Elaboración propia. 2017. 
Forma de evaluación de las prácticas

Cada estudiante presentó un informe final con el diagnóstico y la propuesta de intervención del subsistema designado para cada caso con toda la documentación necesaria y todas las fases anteriormente enunciadas.

\section{Acerca de la Tutoría}

Las actividades de los estudiantes se realizaron bajo la tutoría ad-honorem de docentes pertenecientes al Área de Tecnología, Producción y Gestión (Figura 5).

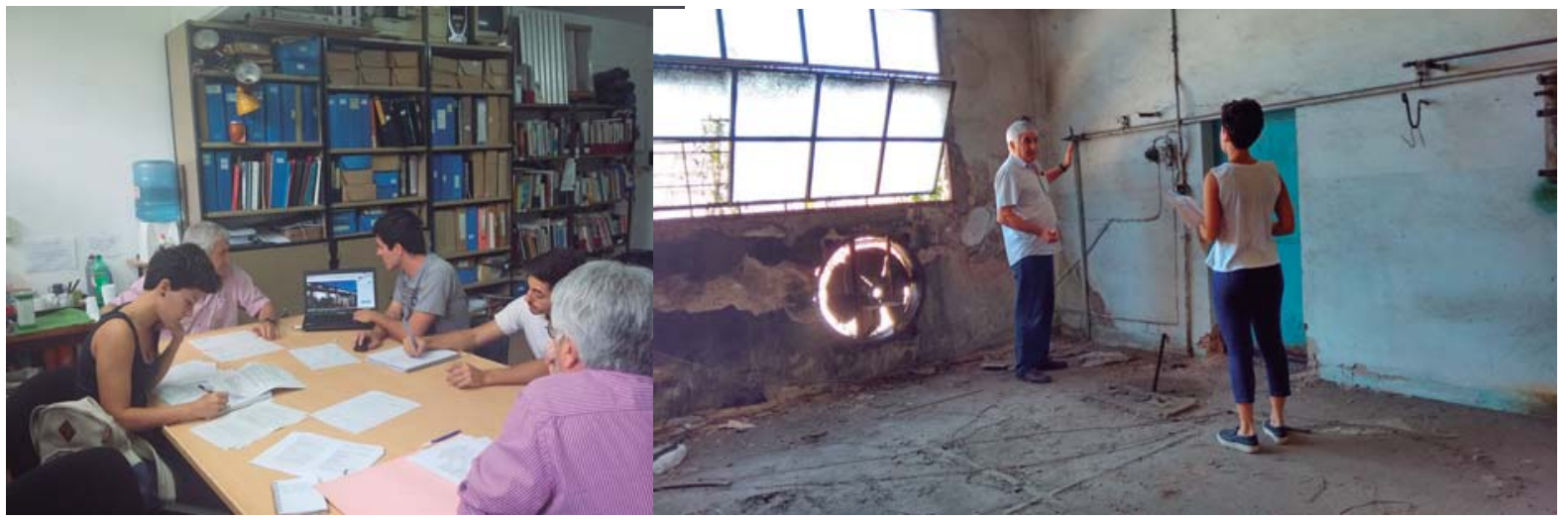

Figura 5. Tutoría de las prácticas pre profesionales asistidas.

Fuente: Elaboración propia. 2017.

\section{$4 \quad$ Resultados Obtenidos}

Tomando como premisa los objetivos de las PPPA se pudieron verificar los conocimientos adquiridos en la currícula académica en el marco de una problemática acotada y bajo el acompañamiento de los tutores para fortalecer la adquisición práctica de los conocimientos formales propios de las incumbencias profesionales, generándose así un pensamiento sistémico integrado para la labor profesional. Es de destacar que este trabajo fue expuesto mediante una presentación formal de los alumnos y tutores frente al Decano de la FAU y ha sido considerado de valioso aporte para la Facultad, capaz de contribuir a elevar el nivel tecnológico de la misma. Es así como estas actividades retroalimentan al curso de grado y visibilizan actividades de vinculación con el medio (Figura 6).

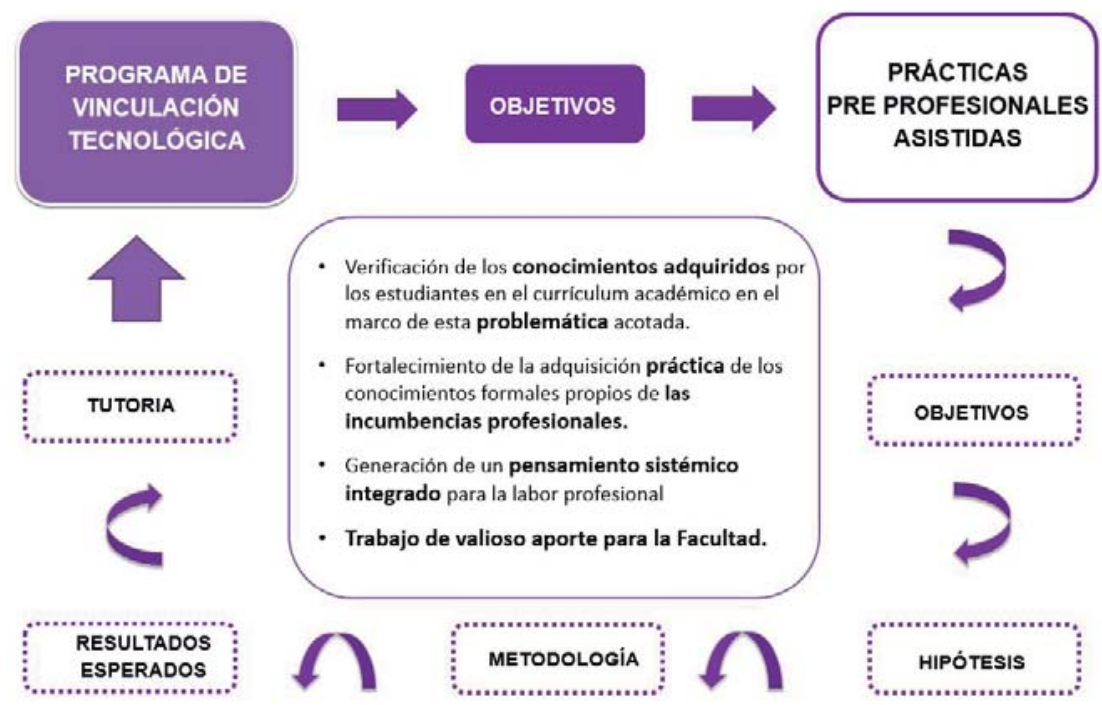

Figura 6. Resultados obtenidos.

Fuente: Elaboración propia. 2017.

La implementación de esta estrategia metodológica en las PPPA promueve el aprendizaje de conocimientos, aptitudes y criterios necesarios como parte del proceso de proyecto, abarcando aspectos constructivos, de programación y de ejecución de la obra, que permiten dar adecuada 
respuesta a la recuperación de edificios, en este caso, universitarios. Se promueve así, a través del diseño constructivo, la programación y la ejecución de la obra, "la adquisición de la sensibilidad y el conocimiento metodológico que posibilite resolver problemas concretos en tiempos ciertos, así como proponer diseños nuevos, económicos y eficaces".

Asimismo, el desarrollo de estas prácticas favoreció la capacidad de comprender la totalidad de los aspectos de la producción de la obra como la decodificación total del proyecto arquitectónico en todos sus niveles de composición teórica, promoviendo la elección de las Tecnologías de Producción de la obra, de acuerdo a la complejidad, ubicación y disponibilidad de recursos económicos para la realización de la misma.

\section{Conclusiones}

La mejor manera de superar el actual proceso de empobrecimiento ético y moral, de dependencia económica, cultural y tecnológica, es haciendo el esfuerzo de transformarse en hombres inteligentes y ayudar a otros en busca de esa misma transformación. El sinceramiento, el ejercicio permanente del pensamiento, la diferenciación entre voluntad y posibilidad, la aceptación de las diferencias ideológicas, la capacidad de integración social, el reconocimiento y cualificación de las prioridades, son sólo algunos de los conceptos que deben estar presentes permanentemente en la Universidad. Interpretar y comprender el pasado que nos da origen, el presente que vivimos y como consecuencia el futuro que esperamos es parte de la mirada concientizadora y crítica que se pretende transmitir.

De este y futuros trabajos surgirán hombres capaces de integrar ideas, contrastar propuestas, evaluarlas y en un proceso profundo de comprensión y convergencia proponer la o las soluciones apropiadas a un problema, sabiendo que éste es sólo parte de un todo más amplio y que, como parte, debe ensamblarse en ese todo. En otras palabras, profesionales comprometidos con la realidad argentina, capaces de resolver los problemas del espacio arquitectónico con capacidad y potencialidades que le permitan asimilar los cambios que la sociedad produce. De esta manera el alumno de hoy se capacitará para ejercitar el mecanismo de abordaje a cualquier problema de los tantos que se plantearán en su desempeño profesional.

Este tipo de estrategias de innovación curricular son pasibles de ser replicadas en otras prácticas en pos de proporcionar al alumno una aproximación a la tarea profesional, afianzando y concluyendo el nivel de preparación logrado en el transcurso de la carrera.

\section{Referencias.}

\subsection{Bibliografía recomendada}

Argentina. Ley 13.059 y Decreto Reglamentario 1.030/2010 de Acondicionamiento higrotérmico de la Provincia de Buenos Aires.

Brundtland, Gro Harlem. Nuestro futuro común. Madrid: Alianza Editorial, 1988.

Chandías, Mario E. y Ramos, José Martín. Cómputos y Presupuestos. Manual para la construcción de edificios con computación aplicada. 19a ed. Buenos Aires: Librería y Editorial Alsina, 2004. 446 p Colegio Oficial de Arquitectos de Madrid. Redacción de informes técnicos sobre el estado de la edificación. (Colección: Guías de Asistencia Técnica). Madrid: Fundación Cultural COAM, 1998. 87 p.

CSCAE. Un Vitruvio ecológico. Principios y práctica del proyecto arquitectónico sostenible. Barcelona: Editorial Gustavo Gili, S. A., 2007. 159 p. (AD+E Arquitectura y Diseño + Ecología) ISBN 978-84-252-2155-2.

Edwards, Brian. Guía básica de la sostenibilidad. $2^{a}$ ed. revisada y ampliada. Barcelona: Editorial Gustavo Gili, SL, 2008. 223 p.

Falabella, María Teresita. Cíclico, preventivo y constante: el mantenimiento edilicio y su relación con la patología constructiva. María Teresita Falabella; con colaboración de: Silvia Stivale. $1^{a}$ ed. Buenos Aires: Nobuko, 2006. 212 p.

Gordín, Eduardo W. Patologías habituales de la construcción. Buenos Aires: Fundación Capacitar, 
2013. 194 p. ISBN 978-987-24272-0-7

Instituto Argentino de Normalización y Certificación (IRAM). Normas 11601, 11602, 11603, 11604,11605 y 11630.

Jourda, Françoise-Hélène. Pequeño manual del proyecto sostenible. Barcelona: Editorial Gustavo Gili, SL, 2012. ISBN 978-84-252-2449-2.

Sosa Griffin, María Eugenia; Siem, Geovanni. Manual de Diseño para Edificaciones Energeticamente Eficientes en el Trópico. Caracas: Instituto de Desarrollo Experimental de la Construcción Facultad de Arquitectura y Urbanismo, 2004 - IDEC/FAU/UCV (IDEC). Disponible en:http://idec. fau.ucv.ve/wordpress/wp-content/uploads/2015/09/manual-de-dise\%C3\%B 1 o.pdf 